\title{
Ontogenic Studies of Gastrointestinal Function. II. Lower Esophageal Sphincter Maturation in Neonatal Beagle Puppies
}

\author{
STEVEN B. SPEDALE, NORMAN W. WEISBRODT AND FRANK H. MORRISS, JR. ${ }^{(33)}$ \\ Houston Perinatal Nutrition Laboratory, Department of Pediatrics, and Department of Physiology, Medical School, \\ The University of Texas Health Science Center at Houston, Houston, Texas, USA
}

\begin{abstract}
Summary
The purpose of the present study was to investigate the postnatal development of lower esophageal spincter (LES) competence in the beagle and to determine the role of gastrin in LES functional ontogeny. Eleven beagle puppies taken from two litters were studied. All puppies were allowed to suckle during the first 3 postnatal wk. In the 4th wk, all puppies were weaned, and by the 5th wk only solid chow was offered. Intraluminal manometric pressure determinations were obtained in unanesthetized, unsedated puppies using twin-lumen, $1.7 \mathrm{~mm}$ OD, $1 \mathrm{~mm}$ ID polyvinyl catheters. A 12-15 min baseline period of intraluminal pressure was monitored, after which the puppies received successive doses of $0.03,0.1,1.0$, and $8.0 \mu \mathrm{g} / \mathrm{kg}$ pentagastrin (PG) SC spaced at 45-min intervals.

Progressive increases in LES pressure both during basal periods and during gastric contractions and in gastric fundus (GF) pressures during gastric contractions occurred during the 1st 5 weeks of life. An increase in pressure gradient between the LES and GF during gastric contraction occurred from birth through 5 wk of age. There was no response to $0.1-8.0 \mu \mathrm{g} / \mathrm{kg}$ PG until postnatal days 5-6 when the LES pressure gradient in the basal state increased in response to each of these doses. There was also no significant response by the LES to PG during gastric contraction until days 11-12 when all doses of PG evoked enhancement of LES pressure.

These studies suggest that an increase in LES-GF intraluminal pressure gradient develops during the initial 2 postnatal wk in this species and that this development does not involve the hormone gastrin. Later, however, the pressure gradient may depend upon both age and gastrin since a two-way analysis of variance indicated that not only is LES pressure affected by postnatal age and by $P G$ dose, but that there is an interaction between postnatal age and PG dose which is significant. By the fifth wk, there was an apparent dose-response effect in which the lower two doses produced increased LES pressure and the larger two doses produced decreased LES pressures.
\end{abstract}

\section{Speculation}

Gastroesophageal reflux (GER) in some infants may occur when the developmental interplay between endogenous gastrin concentration, increasing sensitivity to gastrin by LES, and sensitivity to gastrin by the muscular stomach proceeds abnormally. Alternatively, either the postnatal increase in LES muscle mass or the maturation of a neural pathway that mediates reflex contraction of the LES during gastric contraction, or both, may be delayed or abnormal in some children with GER. A fourth possible abnormality that may permit GER in still other infants is failure to generate the increasing gastric contraction rate and intraluminal pressures observed to occur in normal development, thus permitting delayed gastric emptying.
Functional incompetence of the lower esophageal sphincter (LES) that results in gastroesophageal reflux (GER) is a common problem in infants $(6,15,20,23,24,28,29)$. Healthy term and preterm newborn infants normally regurgitate feedings for several days to several wk after birth, but by two months of age this usually subsides (16). A small \% of all infants without hiatal hernia exhibit symptoms of GER which persist abnormally into later infancy, often necessitating treatment. These events suggest that a maturation of LES function normally occurs in a pattern that accomplishes sphincter competence shortly after 40 wk postconceptual age in human infants. The specific mechanisms that underlie LES maturation in the postnatal period, however, are poorly delineated.

Cohen (5) studied the LES-gastric fundus (GF) intraluminal pressure gradient in the opposum, a species that is markedly underdeveloped at birth, at intervals from day 35 to maturity. He found a progressive rise in the pressure gradient from 4.3 to 28.0 $\mathrm{mm} \mathrm{Hg}$. In strips of LES muscle taken from these animals he demonstrated a parallel increase in responsiveness in vitro to gastrin I per mass of muscle as well as an increase in muscle mass with age. Muscle responsiveness to other stimuli, corrected for muscle weight, did not change with the postnatal age. Thus, Cohen concluded that the lower LES intraluminal pressure gradient in the younger animals was associated with a smaller LES muscle mass and a reduced responsiveness to gastrin.

In a previously reported investigation we examined the postnatal development of gastric motility and GF intraluminal pressures in unsedated, unanesthetized term newborn beagle dogs from the day of birth through weaning at $5 \mathrm{wk}$ (22). Gastric contraction rate and intraluminal pressure increased from birth until the ninth postnatal day. After day 9 , the rate remained fairly constant, but intraluminal pressure continued to increase. Neither rate nor pressure was altered by pentagastrin administration during the first 9 days after birth. After day 9, both rate and intraluminal pressure decreased after $8 \mu \mathrm{g} / \mathrm{kg}$ pentagastrin administration. In addition, pentagastrin administration failed to evoke an acid secretory response until the 9th postnatal day. Thus, in the canine stomach, gastric intraluminal pressure increased with postnatal age, but early postnatal gastric function appeared to be insensitive to gastrin.

The purpose of the present study was to investigate the postnatal development of LES competence in the beagle and to determine the role of gastrin in LES functional ontogeny.

\section{MATERIALS AND METHODS}

Eleven beagle puppies taken from two litters (Laboratory Research Enterprises, Kalamazoo, MI) were studied. The mean ( \pm S.E.) weights of the puppies were $312 \pm 22 \mathrm{~g}$ at birth and $1322 \pm$ $100 \mathrm{~g}$ at $5 \mathrm{wk}$. All puppies were allowed to suckle during the first 3 postnatal wk. In the 4 th wk, all puppies were weaned and by the 
5th wk only solid chow (Purina Lab Canine Diet 5006, Richmond, IN) was offered.

Intraluminal manometric pressure determinations were obtained in unanesthetized, unsedated puppies using twin-lumen 1.7 mm OD, $1 \mathrm{~mm}$ ID polyvinyl catheters (Bard-Parker, Rutherford, $\mathrm{NJ})(22)$. The catheters were $38 \mathrm{~cm}$ long; each had a single $1 \mathrm{~mm}-$ diameter side opening which in the catheter bundle were located $2 \mathrm{~cm}$ apart. Catheters were inserted orogastrically with the catheter openings oriented laterally to assure that successive measurements were obtained from a constant location within the LES to avoid the effect of radial asymmetry if present in the neonatal canine LES (8). Catheters were perfused with distilled water by a Harvard Infusion Pump (model 931, Harvard Apparatus, Millis, MA) at a constant infusion rate of $0.51 \mathrm{ml} / \mathrm{min}$. Pressure transducers (model P23ID, Gould Statham Instruments, Inc. Hato Rey, PR) were interposed in the system. A permanent recording of LES and gastric fundus (GF) pressures was made by a Statham recorder (Model SP2000B) with a chart speed of $1 \mathrm{~mm} / \mathrm{sec}$. Atmospheric pressure was designated as zero pressure. The response rate of the instrumentation was $40 \mathrm{~mm} \mathrm{Hg} / \mathrm{sec}$, a response rate that is sufficiently rapid to record with fidelity the relatively slow neonatal canine stomach and LES intraluminal pressure changes (8).

The position of the LES was determined by 2-3 slow continuous pull-through maneuvers. The proximal opening of the catheter then was positioned and held stationary in the LES as the position of maximum pressure; the distal opening was positioned in the fundus of the stomach.

All puppies were studied on an alternate day protocol for the first 14 postnatal days and again at the end of the third, fourth, and fifth wk. Puppies were removed from the mother $2-4 \mathrm{~h}$ before study. After this fasting period, gastric contents were aspirated, the stomach was rinsed with distilled water, and the twin-lumen catheter was introduced. A 12-15 min baseline period of intraluminal pressure was monitored, after which the puppies received succcessive doses of $0.03,0.1,1.0$, and $8.0 \mu \mathrm{g} / \mathrm{kg}$ pentagastrin (PG) SC (Ayerst Laboratories, NY) spaced at 45-min intervals. After administration of each dose, intraluminal pressures were monitored for $15 \mathrm{~min}$. The stomach was aspirated intermittently to remove the perfused water.

Recordings of intraluminal pressures in the LES and GF were made during both basal and contracting states of the stomach. For each puppy, the basal LES and GF intraluminal pressure values were obtained by averaging respectively the intraluminal pressures at 5 -sec intervals during a 1 -min period in which no contraction of the LES or GF occurred. Contraction pressures for each puppy were obtained by averaging respectively the peak values recorded during at least three consecutive contractions of the GF. Responses to PG were evaluated similarly during a monitoring period of 510 min after administration of each dose of PG. No attempt was made to measure the length of the LES at various postnatal ages.

Standard parametric statistical tests were used (25). Two-way analysis of variance was employed to test the significance of the effects of increasing postnatal age and PG dose on LES pressures. One-way analysis of variance and the Student-Newman-Keuls tests were employed to determine the earliest postnatal age at which each dose of pentagastrin exerted an effect on LES pressure.

\section{RESULTS}

The postnatal development of LES and GF intraluminal pressures in beagle puppies during basal periods and during gastric contractions is illustrated in Figure 1. Progressive increases in LES pressure both during basal periods and during gastric contractions and in GF pressures during gastric contractions occurred during the first $5 \mathrm{wk}$ of life $(P<0.001)$. Basal GF pressure was stable for the first 4 days of life, increased on days 5-6, and remained stable at the higher level for the remainder of the postnatal period investigated.

The pressure gradient between the LES and GF during gastric contractions is shown in Figure 2. An increase in pressure gradient occurred from birth through $5 \mathrm{wk}$ of age $(P<0.00001)$. On days

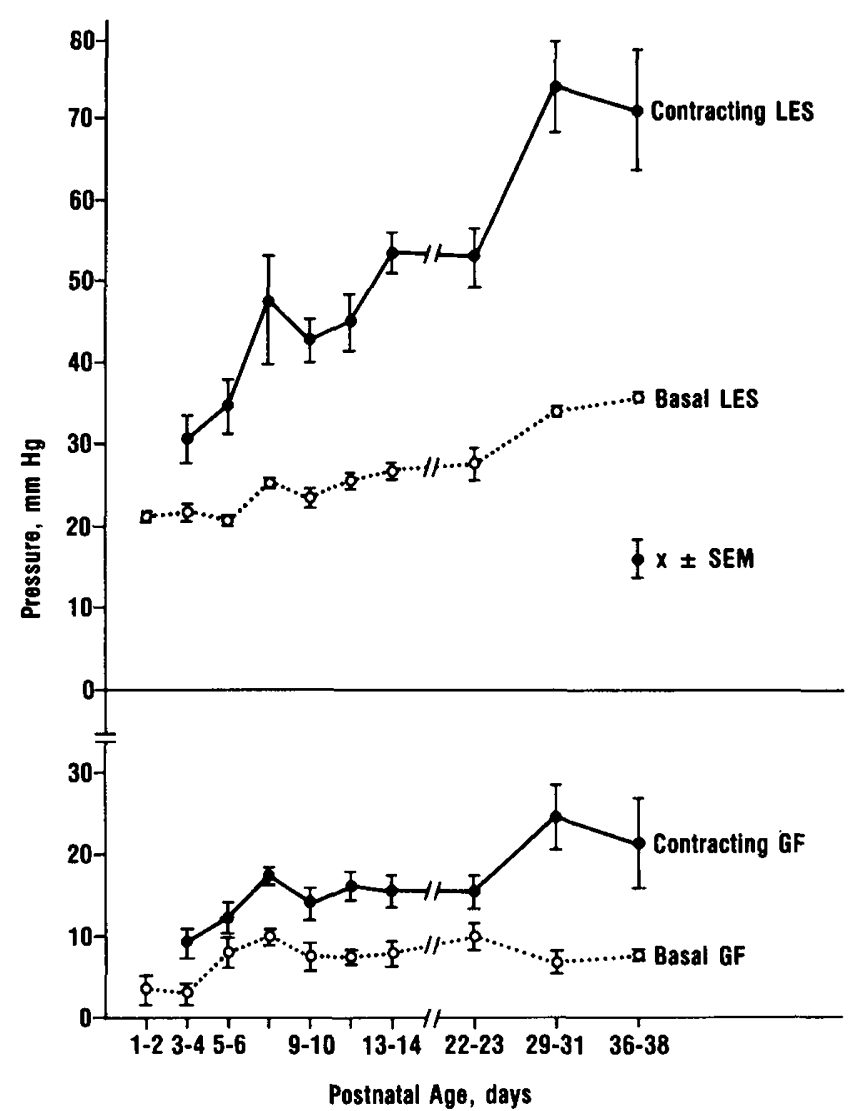

Fig. 1. (Top Panel) Peak intraluminal pressure at the lower esophageal sphincter (LES) during the first 5 postnatal wk in beagle puppies during absence of gastric contractions (basal) and during gastric contractions (contracting). (Bottom Panel) Peak intraluminal pressure in the gastric fundus during the first 5 postnatal wk in beagle puppies during basal and contracting states.

1-2 the gradient in the one puppy which exhibited a gastric contraction was $2.5 \mathrm{~mm} \mathrm{Hg}$. By days 3-4 all puppies experienced gastric contractions with a gradient of $21.4 \pm 3.8 \mathrm{~mm} \mathrm{Hg}$. The gradient subsequently increased to $49.6 \pm 4.0 \mathrm{~mm} \mathrm{Hg}$ by 5 weeks of age. The pressure gradient between basal LES and basal GF also exhibited a similar, but smaller, increase from $17.7 \pm 1.6 \mathrm{~mm}$ $\mathrm{Hg}$ on days $\mathrm{I}-2$ to $28.0 \pm 0.6 \mathrm{~mm} \mathrm{Hg}$ at 5 weeks $(P<0.001)$.

When the stomach was not contracting (basal state), there was no significant change in LES pressure response to the administration of PG at $0.03 \mu \mathrm{g} / \mathrm{kg}$. There was no response to $0.1-8.0 \mu \mathrm{g} / \mathrm{kg}$ PG until postnatal days 5-6 when the LES pressure gradient in the basal state increased by $0.1-1.5 \mathrm{~mm} \mathrm{Hg}$ in response to these doses $(P<0.05)$. There was also no significant response by the LES to PG during gastric contraction until days 11-12 when all doses of PG evoked enhancement of LES pressure $(P<0.05$; (Table 1).

The two-way analysis of variance of LES pressure data during gastric contraction indicated that not only was LES pressure affected by postnatal age and by PG dose, but that there was an interaction between postnatal age and PG dose, which was significant $(P<0.001$; Table 1). This interaction may be visualized in Figure 3, which illustrates the \% change in LES pressure during gastric contraction produced by administration of the four doses of PG at selected postnatal intervals. Beginning on days 11-12, all PG doses evoked significant increases in LES pressure. Later, in the fifth wk, the enhancement of LES pressure evoked by 0.03 and $0.1 \mu \mathrm{g} / \mathrm{kg}$ increased, but the larger two doses, 1.0 and $8.0 \mu \mathrm{g} /$ $\mathrm{kg}$, became inhibitory, decreasing the LES pressure during gastric contraction. By the fifth wk, there was an apparent dose-response effect in which the lowest dose produced the greatest increase in LES pressure, and the largest dose produced the greatest decrease in LES pressure. 


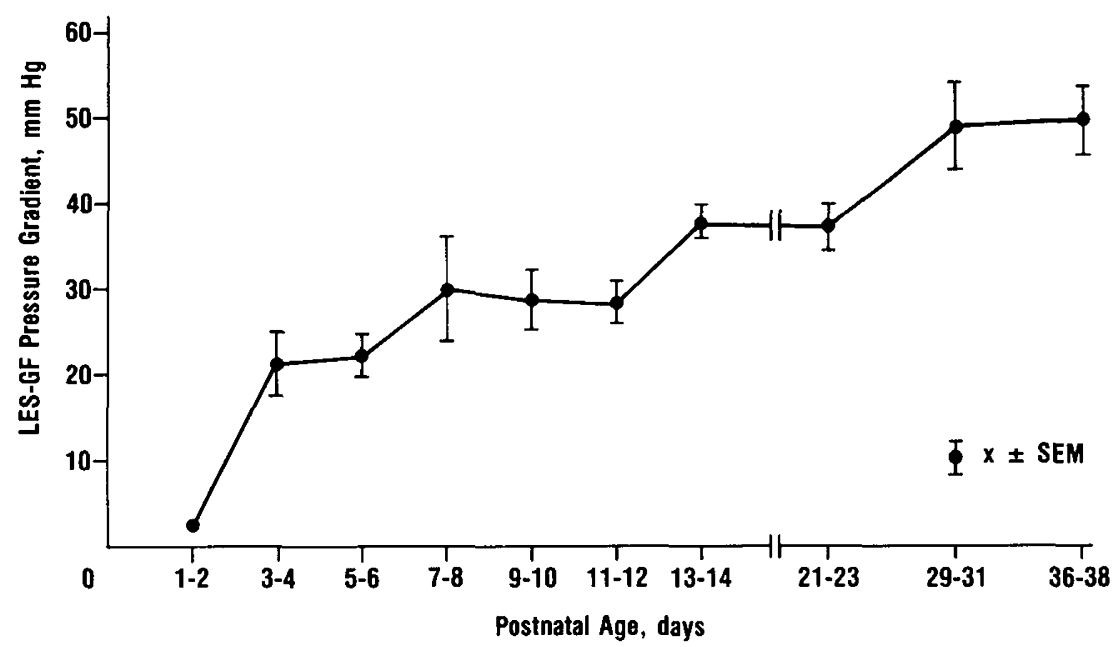

Fig. 2. Intraluminal pressure gradient between lower esophageal sphincter (LES) and gastric fundus (GF) during gastric contractions in beagle puppies during the first 5 postnatal wk.

Table 1. Mean lower esophageal sphincter intraluminal pressures in $\mathrm{mm} \mathrm{Hg}$ obtained during gastric contractions in beagle puppies before pentagastrin administration and after administration of increasing doses of pentagastrin, by postnatal age

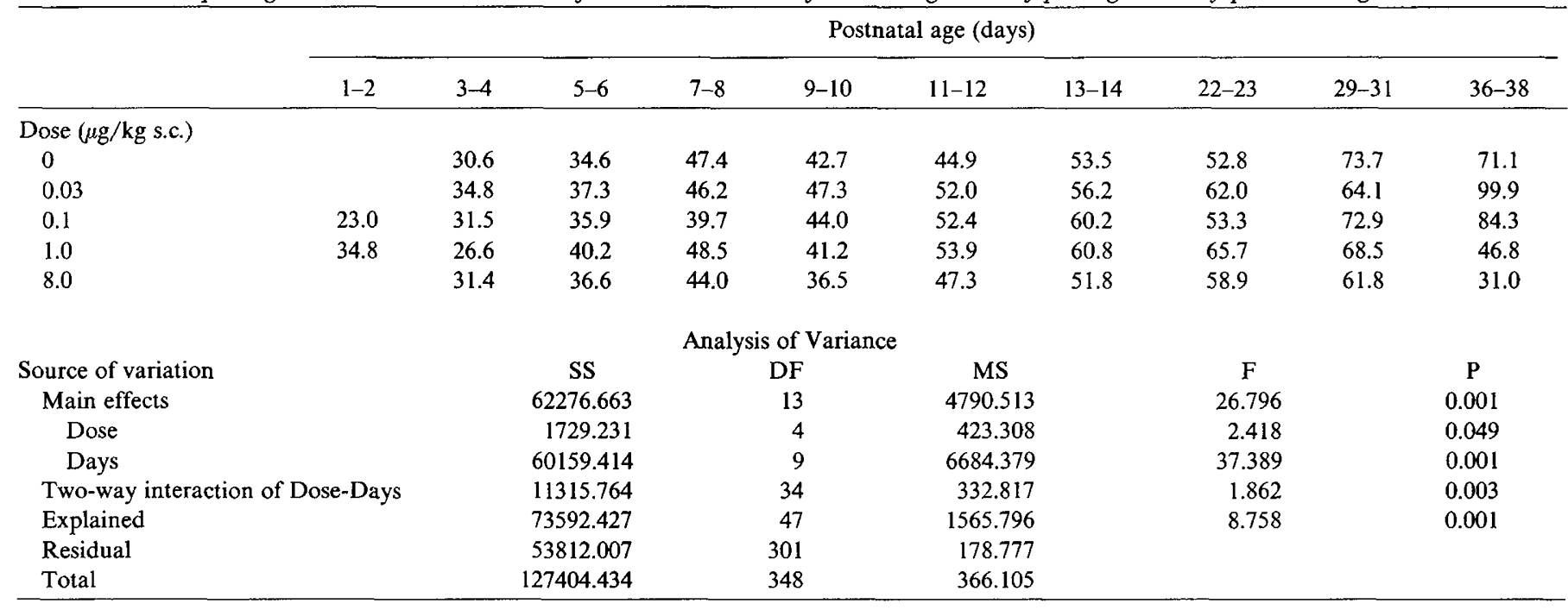

\section{DISCUSSION}

Aspects of the postnatal ontogeny of LES function have been studied by others in human infants as well as in experimental animals. It is important in reviewing those studies and in considering the present study to distinguish whether LES intraluminal pressure was monitored between swallows, during swallowing, in the absence of gastric contractions, or during gastric contractions, as well as to distinguish between LES intraluminal pressure and LES-GF intraluminal pressure gradient.

Strawczynski and co-workers (29) studied normal infants at ages 1-2 wk and 3-6 wk with a manometric technique. They found that the LES-GF intraluminal pressure gradient that was present when the infant was not swallowing was low for the first $2 \mathrm{wk}$ of life, increased during 2-4 wk, and was not different from adult values in 1-month old infants. Older infants who regurgitated had LES-GF intraluminal pressure gradients similar to those found in 1-2-wk-old infants. Gryboski et al. (14) observed that the LES-GF intraluminal pressure gradient in the absence of swallowing and gastric contractions in neonates was essentially absent for 6 days and then began to increase after the sixth day of life. Preterm neonates between 3-7 days postnatal age generated LES-GF intraluminal pressure gradients similar to those of term infants between 1-12 h old (13). Boix-Ochoa and Canals (4) performed esophageal and gastric manometry in preterm and term infants and observed intraluminal pressure increases in both regions with increasing postnatal age. They concluded that an effective antireflux pressure barrier was developed by $5-7 \mathrm{wk}$ after birth. Cohen's (5) studies in the opossum cited above also measured the development of the LES-GF intraluminal pressure gradient in the absence of gastric contractions in anesthetized animals.

The present investigations reveal that the LES of the term newborn beagle puppy generates an increasing intraluminal pressure gradient relevant to the gastric fundus both in the absence of gastric contractions as well as during gastric contractions. In this regard, our results in the unsedated, unanesthetized dog agree with those of Cohen in the anesthetized opossum; in both species, there is an increase in LES intraluminal pressure gradient in the absence of contractions with postnatal age. Moreover, in dogs during the $5 \mathrm{wk}$ after birth, the LES-GF pressure gradient during gastric contractions increases also.

In a previously published study, we described the increase in intragastric pressure during contractions which occur in beagle dogs during the same postnatal period (22). The contraction rate of the stomach also increased during the first 11 postnatal days. Despite the increasing gastric contraction rate and intraluminal pressures that occur during the first wks of postnatal life, we observed in the present study no gastric contraction that was unassociated with a simultaneous elevation in LES intraluminal pressure that was greater than the intragastric pressure. Thus, the 


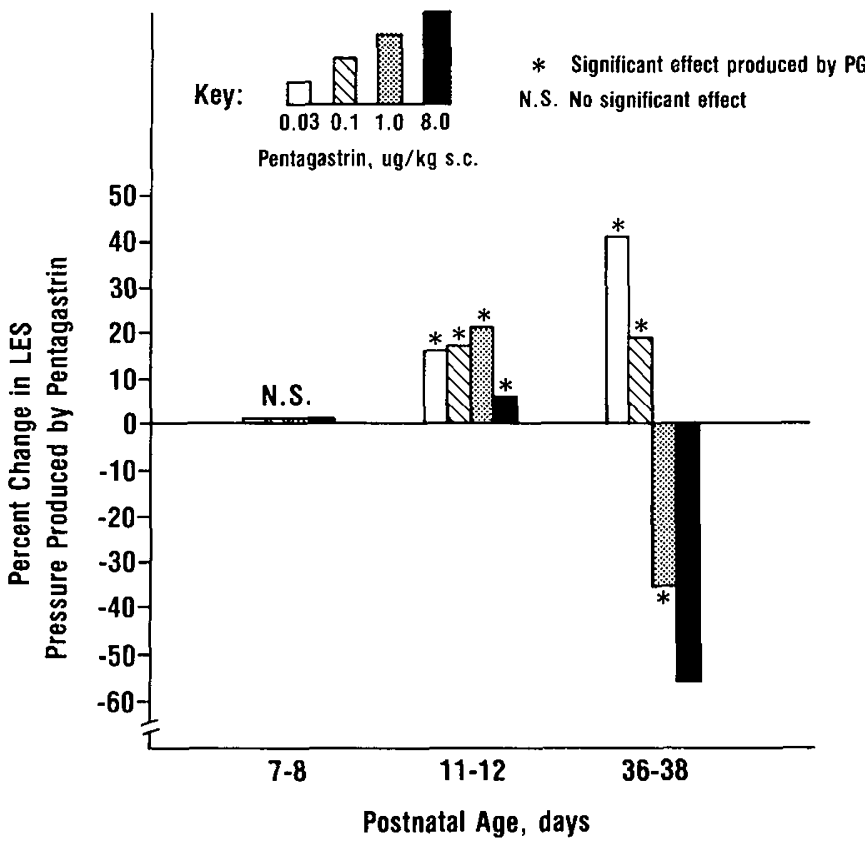

Fig. 3. The \% change in lower esophageal sphincter (LES) intraluminal pressure during gastric contractions that is produced by graded doses of pentagastrin $(\mathrm{PG})$ at different postnatal ages during the first 5 postnatal wk in beagle puppies. These bars graphically represent the interaction of the effects of postnatal age and PG dose on LES pressure which was evaluated statistically by two-way analysis of variance (see "Table 1"). With increasing postnatal age there is increasing LES sensitivity to PG; with increasing postnatal age the smallest dose, $0.01 \mu \mathrm{g} / \mathrm{kg}$ subcutaneously produces increasing enhancement of LES pressure, whereas the larger doses enhance LES pressure initially to a greater degree than the smallest dose, but subsequently produce decreases in LES pressure.

LES functions consistently and competently to prevent reflux in newborn dogs immediately after birth.

The LES was, however, unresponsive during gastric contraction to administered pentagastrin until the middle of the second postnatal wk. This delay in responsiveness to pentagastrin is similar to two observations made previously in the stomach: both the gastric contraction rate and the intragastric $\mathrm{pH}$ were unaffected by $\mathrm{PG}$ administration until the middle of the second wk when PG administration in pharmacologic doses evoked a decrease in contraction rate and $\mathrm{pH}(22)$. Moreover, endogenous serum gastrin concentrations increased in newborn beagles until the middle of the second postnatal wk when values several times adult concentrations were reached (22). Thereafter, serum gastrin concentrations in the puppies decreased. Taken together, these observations support the hypothesis that in this species the LES, as well as the muscular stomach and oxyntic gland, are unresponsive to endogenous gastrin or its administered analogue until the 9-11th day after birth. Thus, the increase in the LES-GF intraluminal pressure gradient that develops during the initial two postnatal weeks is not directly due to gastrin. Consistent with this interpretation of our results in dogs is the lack of correlation between LES intraluminal pressures and serum or plasma gastrin concentrations in human infants. Circulating gastrin levels are greater at birth and in the immediate newborn period than normal adult fasting values and decline as body surface area increases $(10,18,27,31)$; concurrently, LES intraluminal pressure rises $(4,13,14,29)$. The continued increase in pressure gradient after $2 \mathrm{wk}$ in the dog, however, may involve the hormone gastrin as has been postulated by Cohen for the opossum.

Whether the apparent insensitivity to the hormone at these target locations in the dog is a manifestation of reduced hormone receptor number or affinity or of some other factor such as a biologically less active form of hormone or the presence of blocking substances is unknown. The neonatal rat gastric mucosa is also insensitive to gastrin during the preweaning period, and this has been found to be caused by absence of the receptors for the hormone (30). In the rat, mucosal gastrin receptors appear at the normal time for weaning, even if weaning to solid chow is artificially delayed. In fact, the appearance of mucosal gastrin receptors in the rat, like many other changes in rat gastrointestinal function, appears to be mediated by adrenal corticosteroids (26). Whether gastrin receptors in neonatal canine LES, gastric muscle, and oxyntic gland are induced by corticosteroids is unknown, but such an hypothesis may be drawn from the rodent studies to account for these observations in the beagle.

Elucidation of the physiologic events that underlie LES maturation and functional competence during gastric contractions is of interest to physicians who care for infants and children. Not only has the incidence of GER in infants (1:500 livebirths) been found to be greater than previously estimated (15), the list of clinical sequelae of reflux has expanded to include not only vomiting, failure to thrive, esophagitis and stricture formation, but also recurrent pneumonia, chronic asthma, near-miss sudden infant death, apnea, a condition that mimics bronchopulmonary dysplasia, and other manifestations $(3,15,16)$.

In adult animals it has been shown that resting LES pressure is due to an inherent myogenic tone (11). Because muscle mass increases with age, it is likely that the increase in intraluminal pressure is due to muscle mass. On the other hand, the increase in the LES-GF intraluminal pressure gradient during gastric contractions may be due to an enhanced responsiveness of the muscle to various neural inputs. Reflex contraction of the LES occurs with abdominal compression or in association with contractions of the stomach $(7,9,21)$. There is evidence that the increase in LES intraluminal pressure during gastric contraction is mediated by a neural reflex which involves a cholinergic component $(1,21)$. The increase in intraluminal pressure seen in our study, especially the increase in pressure gradient during gastric contractions, could be due to the development of this reflex neural pathway. This is only one possibility of many, however, because the lower esophageal sphincter is sensitive to many neurotransmitters and hormones (12). From the foregoing discussion two possible mechanisms that might produce symptomatic GER may be postulated: delayed or poor increase in muscle mass of the LES and delayed maturation of the neural reflex.

Prolonged monitoring of the esophageal $\mathrm{pH}, 3 \mathrm{~cm}$ proximal to the gastroesophageal junction in 14 asymptomatic term and preterm infants at postnatal ages less than $8 \mathrm{wk}$, has indicated that some reflux was present in all, in a pattern not different from that observed in normal older children (16). A significantly more severe GER pattern was observed in 14 infants who were symptomatic with respiratory stress and apnea (16). These clinical observations suggest that GER is not an all- or- none phenomenon, but that GER becomes symptomatic when it is abnormally frequent or prolonged. Moreover, LES pressure is not consistently decreased in infants with gastroesophageal reflux (2). Recent clinical investigations suggest that delayed gastric emptying, rather than abnormally low LES intraluminal pressure, may be an important feature of severe GER (17). Since reflux is a normal event after eating (19), abnormally prolonged retention of gastric contents may predispose to increased frequency or duration of GER in symptomatic patients. Failure to generate a postnatal pattern of increasing gastric contraction rate and/or intraluminal pressures similar to the pattern demonstrated in normal dogs in our earlier report (22) may underlie the gastric retention of feedings observed in some infants with GER.

The present study of LES function together with our previous report provide evidence that the pressure gradient from LES to gastric fundus during gastric contractions increases rapidly in normally developing puppies, and that this maturational process appears to proceed initially independently of gastrin. After the second postnatal wk, however, elevated endogenous gastrin levels may contribute to the development of the LES-GF gradient. Thus, there is a postnatal interplay between endogenous gastrin concentration, LES sensitivity to gastrin, and GF sensitivity to gastrin. 
Abnormal development of this interplay is a fourth possible pathophysiologic mechanism which might lead to GER in infants.

\section{REFERENCES AND NOTES}

1. Angorn, I. B., Dimopoulos, G., Hegarty, M. M., and Moshal, M. G.: The effect of vagotomy on the lower esophageal sphincter: a manometric study. Br. J. Surg., 64: 466 (1977)

2. Arasu, T. S., Wyllie, R., Fitzgerald, J. F., Franken, E. A., Siddiqui, A. R., Lehman, G. A., Eigen, H., and Grosfeld, J. L.: Gastroesophageal reflux in infants and children-comparative accuracy of diagnostic methods. J. Pediatr. 96: 798 (1980).

3. Berquist, W. E., Rachelefsky, G. S., Kadden, M., Siegel, S. C., Katz, R. M. Fonkalsrud, E. W., and Ament, M. E.: Gastroesophageal reflux-associated recurrent pneumonia and chronic asthma in children. Pediatrics, 68: 29 (1981)

4. Boix-Ochoa, J. and Canals, J.: Maturation of the lower esophagus. J. Pediatr. Surg., 11: 749 (1976).

5. Cohen, S.: Developmental characteristics of lower esophageal sphincter function: a possible mechanism for infantile chalasia. Gastroenterology, 67: 252 (1974).

6. Darling, D. B., Fisher, J. H., and Gellis, S. S.: Hiatal hernia and gastroesophageal reflux in infants and children: analysis of the incidence in North American children. Pediatrics, 54: 450 (1974).

7. Diamant, N. E. and Akin, A. N.: Effect of gastric contractions on the lower esophageal sphincter. Gastroenterology, 63: 38 (1972).

8. Dodds, W. J.: Instrumentation and methods for intraluminal esophageal manometry. Arch Intern. Med., 136: 515 (1976).

9. Dodds, W. J., Hogan, W. J., Miller, W. N., Stef, J. J., Arndorfer, R. C., and Lydon, S. B.: Effect of increased intra-abdominal pressure on lower esophageal sphincter pressure. Am. J. Dig. Dis., 20: 298 (1975).

10. Euler, A. R., Ament, M. E., and Walsh, J. H.: Human newborn hypergastrinemia: an investigation of prenatal and perinatal factors and their effects on gastrin Pediatr. Res., 12: 652 (1978).

11. Goyal, R. K. and Rattan, S.: Genesis of basal sphincter pressure: effect of tetrodotoxin on lower esophageal sphincter pressure in opossum in vivo. Gastroenterology, 71: 62 (1976).

12. Goyal, R. K. and Rattan, S.: Neurohumoral, hormonal, and drug receptors for the lower esophageal sphincter. Gastroenterology, 74: 598 (1978).

13. Gryboski, J. D.: The swallowing mechanism of the neonate. I. Esophageal and gastric motility. Pediatrics, 35: 445 (1965)

14. Gryboski, J. D., Thayer, W. R., and Spiro, H. M.: Esophageal motility in infants and children. Pediatrics, 31: 382 (1963).

15. Herbst, J. J.: Gastroesophageal reflux. J. Pediatr., 98: 859 (1981).

16. Herbst, J. J., Minton, S. D., and Book, L. S.: Gastroesophageal reflux causing respiratory distress and apnea in newborn infants. J. Pediatr., 95: 763 (1979).
17. Hillemeier, A. C., Lange, R., McCallum, R., Seashore, J., and Gryboski, J.: Delayed gastric emptying in infants with gastroesophageal reflux. J. Pediatr. 98: 190 (1981).

18. Janik, J. S., Akbar, A. M., Burrington, J. D., and Burke, G.: Serum gastrin levels in infants and children. Pediatrics, 60:60 (1977).

19. Jolley, S. G., Johnson, D. G., Herbst, J. J., Alberto Pena, R., and Garnier, R.: An assessment of gastroesophageal reflux in children by extended $\mathrm{pH}$ monitoring of the distal esophagus. Surgery, 84: 16 (1978).

20. Keitel, H. G. and Ziegra, S. R.: Regurgitation in the full-term infant: a controlled clinical study. Am. J. Dis. Child., 102: 749 (1961).

21. Lind, T. F., Crispin, J. S., and McIver, D. K.: The effect of atropine on the gastroesophageal sphincter. Can. J. Physiol. Pharmacol., 46: 233 (1968).

22. Malloy, M. H., Morriss, F. H., Denson, S. E., Weisbrodt, N. W., Lichtenberger, L. M., and Adcock, E. W.: Neonatal gastric motility in dogs: maturation and response to pentagastrin. Am. J. Physiol., 236: E562 (1979).

23. Moroz, S. P., Espinoza, J., Cumming, W. A., and Diamant, N. E.: Lower esophageal sphincter function in children with and without gastroesophageal reflux. Gastroenterology, 71: 236 (1976).

24. Neuhauser, E. B. D. and Berenberg, W.: Cardio-esophageal relaxation as a cause of vomiting in infants. Radiology, 48: 480 (1947).

25. Nie, N. H., Hull, C. H., Jenkins, J. G., Steinbrenner, K., and Bent, D.: Statistical Package for Social Sciences. Ed. 2, p. 398 (New York, McGraw-Hill Book Co., 1975).

26. Peitsch W., Takeuchi, K., and Johnson, L. R.: Mucosal gastrin receptor. VI. Induction by corticosterone in newborn rats. Am. J. Physiol., 240: G442 (1981).

27. Sann, L., Chayvialle, Bremond, A., and Lambert, R.: Serum gastrin level in early childhood. Arch. Dis. Child., 50: 782 (1975).

28. Sondheimer, J. M. and Morris, B. A.: Gastroesophageal reflux among severely retarded children. J. Pediatr., 94: 710 (1979).

29. Strawczynski, H., Beck, I. T., McKenna, R. D., and Nickerson, G. H.: The behavior of the lower esophageal sphincter in infants and its relationship to gastroesophageal regurgitation. J. Pediatr., 64: 17 (1964).

30. Takeuchi, K., Peitsch, W., and Johnson, L. R.: Mucosal gastrin receptor. V. Development in newborn rats. Am. J. Physiol., 240: G163 (1981).

31. von Berger, L., Henrichs, I., Raptis, S., Heinze, E., Jonatha, W., Teller, W. M., and Pfeiffer, E. F.: Gastrin concentration in plasma of the neonate at birth and after the first feeding. Pediatrics, 58: 264 (1976)

32. The authors are grateful to Mrs. Sherry Walker for her invaluable assistance with the statistical analysis of the data presented.

33. Requests for reprints should be addressed to: Dr Frank H. Morriss, Jr., Department of Pediatrics, University of Texas Medical School at Houston, 6431 Fannin, Houston, TX 77030.

34. This research was supported by HHS Grant \#1 P01 HD 13021.

35. Received for publication October 21, 1981.

36. Accepted for publication March 15, 1982. 